\title{
Integration of gaze information during online language comprehension and learning
}

\author{
Kyle MacDonald (kemacdonald@ucla.edu) \\ Department of Communication, UCLA \\ Elizabeth Swanson (elizswan@stanford.edu) \\ Department of Psychology, Stanford University \\ Michael C. Frank (mcfrank@stanford.edu) \\ Department of Psychology, Stanford University
}

\begin{abstract}
Face-to-face communication provides access to visual information that can support language processing. But do listeners automatically seek social information without regard to the language processing task? Here, we present two eye-tracking studies that ask whether listeners' knowledge of word-object links changes how they actively gather a social cue to reference (eye gaze) during real-time language processing. First, when processing familiar words, children and adults did not delay their gaze shifts to seek a disambiguating gaze cue. When processing novel words, however, children and adults fixated longer on a speaker who provided a gaze cue, which led to an increase in looking to the named object and less looking to the other object in the scene. These results suggest that listeners use their knowledge of object labels when deciding how to allocate visual attention to social partners, which in turn changes the visual input to language processing mechanisms.
\end{abstract}

Keywords: eye movements; language processing; information-seeking; word learning; gaze following

\section{Introduction}

Understanding language in real-time is hard. Consider that even in grounded language comprehension, people can talk about many things, with no guarantee that they use familiar words. This flexibility creates the potential for a speaker's intended meaning to be mostly unconstrained. Listeners, however, can overcome ambiguity by integrating visual information available in face-to-face communication (e.g., the direction of a speaker's gaze) that constrains the interpretation of an utterance (Vigliocco, Perniss, \& Vinson, 2014). But do listeners strategically seek supportive visual information from other speakers? And does this information seeking depend on the listener's uncertainty over word meaning?

Prior empirical and theoretical work on language processing, shows that listeners integrate input from multiple sources to constrain the set of possible interpretations of an utterance (see McClelland, Mirman, and Holt, 2006, for a review). For example, adults are faster to process information and make fewer comprehension errors if a speaker provides gestures with redundant cues to meaning (Kelly, Özyürek, \& Maris, 2010). Moreover, developmental research shows that parents actively use visual cues such as gestures and direction of their gaze to structure language interactions with their children (Estigarribia \& Clark, 2007). And even 16-month-olds can use the direction of another speaker's gaze to infer the meaning of a new word (Baldwin, 1993).
These findings suggest that visual information can facilitate language processing. The visual signal, however, is transient and the value of fixating on a communicative partner can vary depending on the language processing task. Thus, rather than randomly fixate a scene, listeners might strategically deploy their fixations to informative locations that maximize successful comprehension. For example, in prior work, we found that children and adults fixated longer on a speaker's face when processing familiar words in a "noisy" auditory environment, suggesting that they compensated for uncertainty in the auditory signal by gathering more visual information (MacDonald, Marchman, Fernald, \& Frank, 2018). Moreover, recent theoretical and empirical work suggests that children and adults handle noise in the signal by integrating what they perceive with their prior beliefs about the speaker's intended meaning (Fourtassi \& Frank, 2017; Gibson, Bergen, \& Piantadosi, 2013; Yurovsky, Case, \& Frank, 2017).

Here, we pursue the idea that the value of fixating on another speaker could vary as a function of (1) the helpfulness of the interlocutor and (2) the listeners' prior experience with a word-object link. In two experiments, we manipulate whether a speaker provides a valid visual cue to reference: by directing her eye gaze to an object while either producing a concrete, familiar word ("ball") or a novel word ("dax"). We chose this behavior as a case study of active information seeking because gaze following is thought to be relevant for the ecological task of linking language to the world and recent work has found a reliable link between sustained visual attention on objects and word learning (Smith \& Yu, 2013).

Our second goal is to test whether children and adults would show a similar pattern of adapting the timing of their gaze shifts to gather a social cue to reference. It could be that children rely more on visual information from social partners because they have partial knowledge of word-object links and are developing a language model. Moreover, adults have more prior experience with the familiar words in our study and stronger novel word learning skills. Both of these features could diminish the usefulness of seeking a social cue when they process familiar or novel words.

We characterize eye movements as a series of information seeking decisions that aim to minimize uncertainty about the external world (Hayhoe \& Ballard, 2005). Under this account, listeners should consider the utility of fixating on a speaker for achieving their current task goal. We hypoth- 


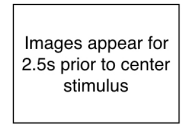

(B)
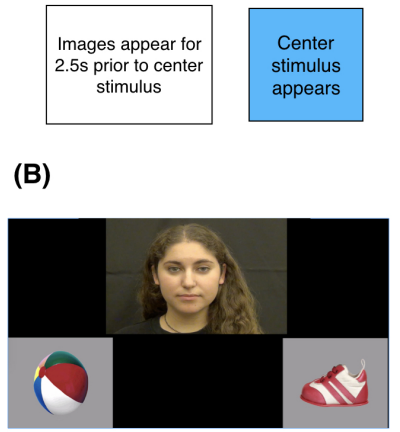

imulus

appears
(C)

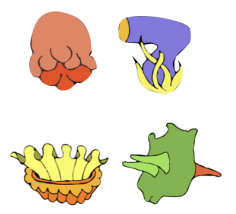

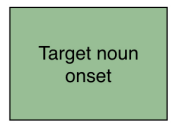
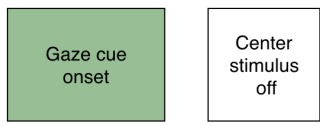

(D) gaze cue
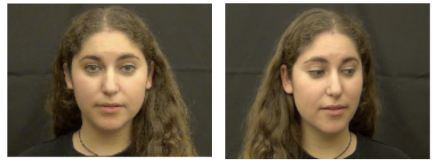

Figure 1: Stimuli for Experiments 1 and 2. Panel A shows the structure of the linguistic stimuli for a single trial. Panel B shows the layout of the fixation locations for all tasks: the center stimulus, the target, and the distracter. Panel C shows a sample of the images used as novel objects in Experiment 2. Panel D shows an example of the social gaze manipulation.

esized that when a communicative partner produces a gaze cue, they become more valuable for the task of disambiguating reference. Our key behavioral prediction is that listeners will delay generating an eye movement away from a speaker's face until seeing where she directs her gaze. This delay will lead to an increase in fixations to the named object.

\section{Experiment 1}

In Experiment 1, we measured the time course of children and adults' decisions about visual fixation as they processed sentences with familiar words ("Where's the ball?"). We manipulated whether the speaker produced a post-nominal gaze cue to the named object. The visual world consisted of three fixation targets (a center video of a person speaking and a target/distracter picture). The primary question of interest was whether listeners would delay shifting their gaze away from the speaker's face if she was likely to generate a gaze cue. We predicted that fixating longer on the speaker would allow listeners to gather more language-relevant visual information to facilitate comprehension. In contrast, if listeners show parallel gaze dynamics across conditions, this would suggest that hearing the familiar word was the primary factor driving shifts in visual attention.

\section{Analytic approach}

To quantify evidence for our predictions, we present two analyses. First, we analyze the time course oflooking to each area of interest (AOI). This measure reflects the mean proportion of trials on which participants fixated on the speaker, the target, or the distracter at every 33-ms interval of the stimulus sentence. We tested condition differences in the proportion looking to the language source using a nonparametric clusterbased permutation analysis, which accounts for the issue of taking multiple comparisons across many time bins in the timecourse (Maris \& Oostenveld, 2007). A higher proportion
Table 1: Age distributions of children in Experiments 1 and 2. All ages are reported in months.

\begin{tabular}{lcccc}
\hline Experiment & $\mathrm{n}$ & Mean & Min & Max \\
\hline Exp. 1 (familiar words) & 38 & 55.50 & 35.60 & 71.04 \\
Exp. 2 (novel words) & 54 & 52.60 & 36.26 & 70.94 \\
\hline
\end{tabular}

of looking to the language source in the gaze condition would indicate listeners' prioritization of seeking visual information from the speaker.

Next, we analyzed the RT and Accuracy of participants' initial gaze shifts away from the speaker to the objects in the scene. RT corresponds to the latency of shifting gaze from the central stimulus to either object measured from the onset of the target noun. All RT distributions were trimmed to between zero and two seconds, and RTs were modeled in log space. Accuracy corresponds to whether participants' first gaze shift landed on the target or the distracter object. If listeners generate slower and more accurate shifts, this provides evidence that gathering more visual information from the speaker led to more robust language processing.

We used the brms (Bürkner, 2017) package to fit Bayesian mixed-effects regression models. The mixed-effects approach allowed us to model the nested structure of our data - multiple trials for each participant and item, and the withinparticipants manipulation. We used Bayesian estimation to quantify uncertainty in our point estimates, which we communicate using a 95\% Highest Density Interval (HDI), providing a range of credible values given the data and model.

\section{Methods}

Participants Participants were native, monolingual English-learning children $(n=38 ; 19 \mathrm{~F})$ and adults $(n=33$; 

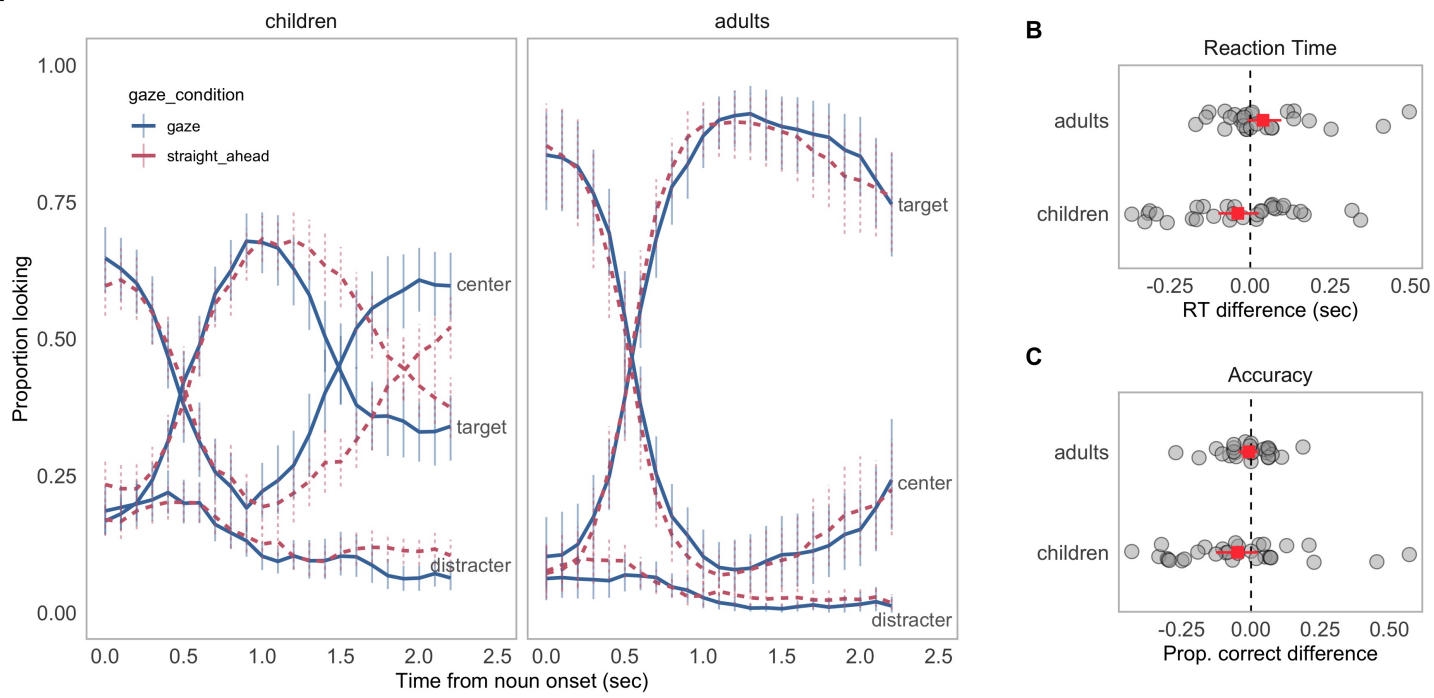

C

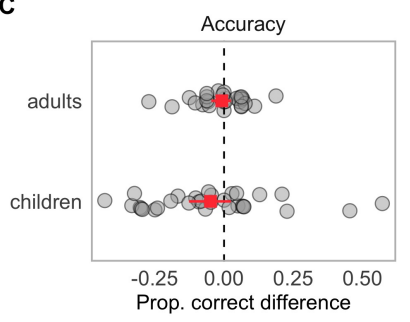

Figure 2: Timecourse looking, first shift Reaction Time (RT), and Accuracy results for children and adults in Experiment 1. Panel A shows the overall looking to the center, target, and distracter stimulus for each gaze condition and age group. Panel B shows the distribution of pairwise contrasts between each participant's RT in the gaze and no-gaze conditions. The square point represents the group means. The vertical dashed line represents the null model of zero condition difference. Error bars represent the 95\% HDI. Panel C shows the same information but for first shift accuracy.

23 F). All participants had no reported history of developmental or language delay and normal vision. 12 participants ( 9 children, 3 adults) were run but not included because either the eye tracker failed to calibrate ( 8 children, 2 adults) or the participant did not complete the task ( 1 children, 1 adults).

Materials Linguistic stimuli. The video/audio stimuli were recorded in a sound-proof room and featured two female speakers who used natural child-directed speech and said one of two phrases: "Hey! Can you find the (target word)" or "Look! Where's the (target word). The target words were: ball, bunny, boat, bottle, cookie, juice, chicken, and shoe. The target words varied in length (shortest $=412 \mathrm{~ms}$, longest $=780 \mathrm{~ms}$ ) with an average length of $587 \mathrm{~ms}$.

Gaze manipulation. To create the stimuli in the gaze condition, the speaker waited until she finished producing the target sentence and then turned her head to gaze at the bottom corner of the camera frame. After looking at the named object, she then returned her gaze to the center of the frame. We chose to allow the length of the gaze cue to vary to keep the stimuli naturalistic. The average length of gaze was 2.12 seconds with a range from 1.78 to 3.07 seconds.

Visual stimuli. The image set consisted of colorful digitized pictures of objects presented in fixed pairs with no phonological overlap between the target and the distracter image (cookie-bottle, boat-juice, bunny-chicken, shoe-ball). The side of target was counterbalanced across trials.

Procedure Participants viewed the task on a screen while their gaze was tracked using an SMI RED corneal-reflection eye-tracker mounted on an LCD monitor, sampling at $30 \mathrm{~Hz}$. The eye-tracker was first calibrated for each participant using a 6-point calibration. On each trial, participants saw two images of familiar objects on the screen for two seconds before the center stimulus appeared. Next, they processed the target sentence followed by two seconds without language to allow for a response. Both children and adults saw 32 trials (16 gaze trials; 16 no-gaze trials) with several filler trials interspersed to maintain interest. The gaze manipulation was blocked with the order of block counterbalanced across participants.

\section{Results and Discussion}

Timecourse looking We first analyzed how the presence of gaze influenced listeners' distribution of attention across the three fixation locations. At target-noun onset, listeners tended to look more at the speaker than the objects. As the target noun unfolded, the mean proportion looking to the center decreased as participants shifted their gaze to the images. After looking to the named referent, listeners tended to shift their gaze back to the speaker's face.

We did not see evidence that the presence of a post-nominal gaze cue changed how listeners allocated attention early in the target word. Children in the gaze condition, however, tended to shift their focus back to the speaker sooner after fixating on the named object, spending more time looking at the speaker throughout the trial $(p<.001)$.

First shift RT and Accuracy Both children and adults generated similar RTs in the gaze (children $M_{r t}=563 \mathrm{~ms}$, adults $M_{r t}=652 \mathrm{~ms}$ ) and no-gaze (children $M_{r t}=576 \mathrm{~ms}$, adults $M_{r t}=608 \mathrm{~ms}$ ) conditions, with the null value falling within 
the 95\% HDI $(\beta=-0.36,[-0.89,0.06])$. Next, we fit the same model to estimate first shift accuracy. Adults generated more accurate gaze shifts $(M=0.9)$ compared to children $(M=$ $0.64)$ with the null value falling outside the $95 \% \mathrm{HDI}\left(\beta_{\text {age }}=\right.$ $-1.76,[-2.19,-1.34])$. Similar to the RT analysis, we did not find evidence of a difference in performance across the gaze conditions $(\beta=0.10,[-0.18,0.41])$.

The time course and first shift analyses suggest that hearing a familiar noun was sufficient for both adults and children to shift visual attention away from the speaker to seek a named referent. Neither age group showed evidence of delaying eye movements to gather a social cue to reference. Children, however, did allocate more attention to the speaker after processing the familiar noun. While we did not predict these results, this behavior seems reasonable if eye movements during familiar language processing are highly-practiced visual routines such that seeking a post-nominal gaze cue becomes less-relevant for disambiguating reference.

The results of Experiment 1 suggest that listeners do not automatically seek social information when it is available and without regard to the current language processing task; instead, it could be that they take uncertainty into account and seek additional social information when ambiguity is higher. This interpretation raises an interesting question: Would listeners adapt the timing of their gaze patterns to gather social information when they do not already know the meaning of a word? That is, when surrounded by unfamiliar objects, the value of fixating on a social partner may increase since this action could provide access to useful disambiguating information such as eye gaze - an idea emphasized by socialpragmatic theories of language acquisition (Bloom, 2002).

\section{Experiment 2}

Experiment 2 explores whether learners will adapt the timing of gaze shifts to seek information from social partners when encountering a novel word. We ask three research questions: (1) do listeners adapt their gaze to seek social information in the context of processing novel words? (2) Does social information seeking change as a function of gaining more exposures to a word-object association? And (3) does following a gaze cue enhance the learning of a novel word-object link? To answer these questions, we compared the timing and accuracy of eye movements during a real-time cross-situational word learning task where participants processed sentences containing a novel word ("Where's the dax?").

We predicted that the presence of gaze would increase participants' looking to a speaker, leading to a higher proportion of fixations to the social target and slower first shift reaction times to the objects. This prediction translates to a main effect of gaze condition on proportion looking to the speaker and on first shift RTs. We also predicted a trial number by gaze condition interaction such that the decrease in RT will be larger on exposure trials in the gaze condition, reflecting a reduction in the need to seek social information after learning the word-object mappings. Finally, we predicted that the presence of gaze would lead to faster learning of the novel word-object links, which would result in more accurate first shifts, faster RTs, and a higher proportion looking to the target object on test trials in the gaze condition.

\section{Methods}

Participants Participants were native, monolingual English-learning children $(n=54 ; 30 \mathrm{~F})$ and adults $(n=30$; $20 \mathrm{~F}$ ). All participants had no reported history of developmental or language delay and normal vision. 6 adults were run but not included because they were not native speakers of English. 7 children participants were run but not included in the analysis because the participant did not complete more than half of the trials in the task.

Materials Linguistic stimuli. The video/audio stimuli were recorded in a sound-proof room and featured two female speakers who used natural child-directed speech and said one of two phrases: "Hey! Can you find the (novel word)" or "Look! Where's the (novel word). The target words were four pseudo-words: bosa, modi, toma, and pifo. The novel words varied in length (shortest $=472.00 \mathrm{~ms}$, longest $=736.00 \mathrm{~ms}$ ) with an average length of $606.31 \mathrm{~ms}$.

Gaze manipulation. The gaze manipulation was identical to Experiment 1. The average length of gaze was $2.06 \mathrm{sec}-$ onds with a range from 1.74 to 2.67 seconds.

Visual stimuli. The image set consisted of 28 colorful digitized pictures of objects that were selected such that they would be interesting to and that children would be unlikely to have already a label associated with the objects. The side of the target picture was counterbalanced across trials.

Procedure The procedure was identical to Experiment 1. Participants watched a series of ambiguous word learning events organized into pairs of one exposure and one test trial. On each trial, participants saw a set of two unfamiliar objects and heard one novel word. Each word occurred in a block of four exposure-test pairs for a total of eight trials for each novel word. Exposure trials in the gaze condition included a gaze cue. Test trials in both conditions did not include a gaze.cue.On each trial within a learning block, one of the objects in the set had consistently appeared on the previous trials (target object), while the other object was a randomly generated novel object that had not been shown in the experiment (distracter object).

\section{Results and Discussion}

Prediction 1: The presence of gaze increases social information seeking. Our primary question of interest was whether listeners would seek a post-nominal gaze cue when processing novel words. In line with our prediction, we found evidence that both children and adults spent more time fixating on a speaker when she provided a social cue to reference (Fig. 3A $\beta_{\text {gaze }}=0.09[0.16,0.01]$ ). Moreover, both children (Gaze $M_{r t}=1,136.77 \mathrm{~ms}$, No-gaze $M_{r t}=878.37 \mathrm{~ms}$ ) and adults (Gaze $M_{r t}=878.65 \mathrm{~ms}$, No-gaze $M_{r t}=726.99 \mathrm{~ms}$ ) had slower RTs in the gaze condition $\left(\beta_{\text {gaze }}=-0.20\right.$, $[-0.38$, 
(A) Looks to speaker

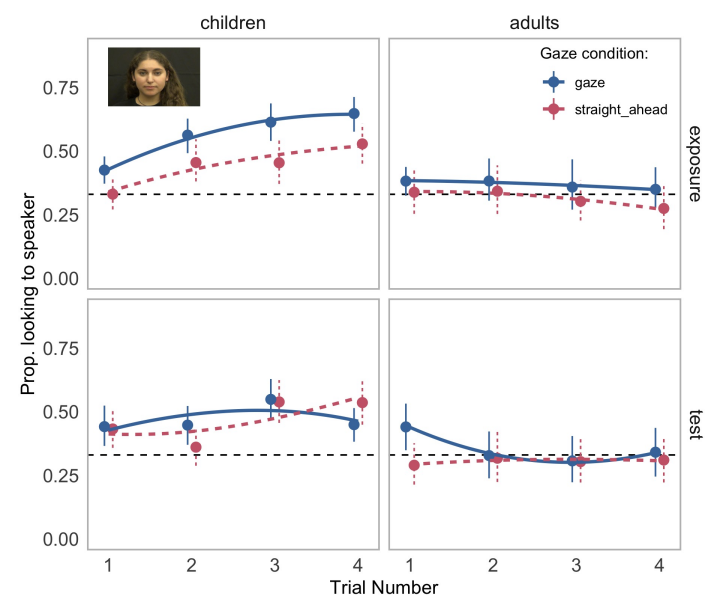

(B) Looks to target object

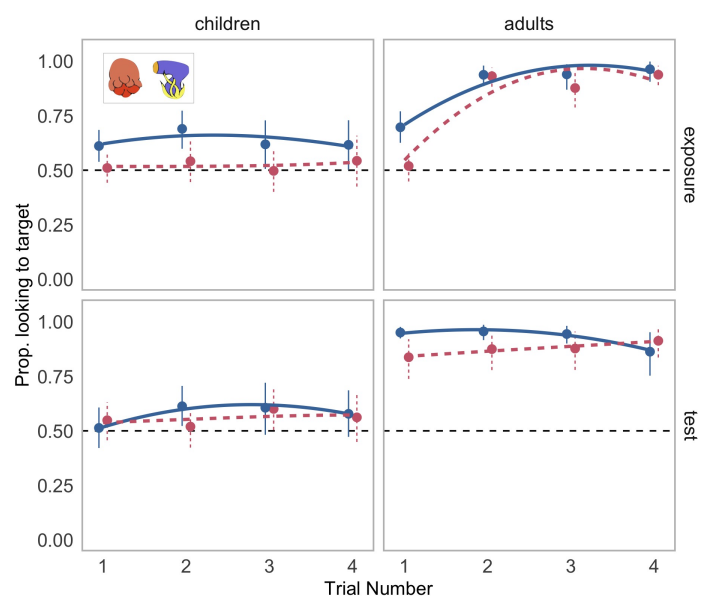

Figure 3: Panel A shows participants' tendency to look at the speaker on exposure and test trials as a function of the trial number within a learning block. The horizontal, dashed line represents the tendency to distribute attention equally across the three AOIs. Color indicates gaze condition and error bars represent $95 \%$ credible intervals. Panel B shows the same information but for target and distracter looking across the learning block.

$-0.01])$, with no evidence of an interaction between gaze condition and age group $\left(\beta_{\text {age } * \text { gaze }}=0.27,[0.11,0.44]\right)$. This result provides support for our key prediction that listeners would adapt their gaze to seek social information when uncertainty over the meaning of words was higher.

Prediction 2: The tendency to fixate on a speaker decreases faster when learning from gaze. We predicted that RTs on exposure trials in the gaze condition would decrease at a faster rate, reflecting a reduction in the need to seek social information after being exposed to consistent wordobject pairings. In contrast to our prediction, we found a developmental difference such that children, but not adults, were more likely to increase their tendency to fixate on the speaker throughout the learning block (Fig 3A, $\beta_{\text {age*trnum }}=$ $0.07,[-0.11,-0.04])$. This developmental difference suggests that looking to a social partner may have been more useful for children who were still trying to disambiguate the novel words; whereas adults showed evidence of successful disambiguation after the second exposure trial and could focus attention on the objects instead.

Prediction 3: Faster learning of novel words from gaze cues. Both children and adults showed evidence of learning the novel word-object links by the end of the task, with the null value of 0.5 falling below the lower bound of the lowest credible interval for children's target looking in the No-gaze context (95\% HDI [0.51, 0.60]). Looking to the target increased as listeners were exposed to more word-object pairings during the task $\left(\beta_{\text {tr.num }}=0.16,[0.09,0.24]\right)$ and was higher when the novel word was accompanied by a gaze cue $\left(\beta_{\text {gaze }}=0.14,[0.21,0.06]\right)$.

Visual inspection of the top row of Fig $3 \mathrm{~B}$ shows that on the first exposure trial, both adults and children used the gaze cue to disambiguate reference, fixating more on the target in the gaze condition. In contrast, adults target looking reached ceiling for both the gaze and no-gaze conditions by trial number two, indicating that they had successfully used co-occurrence information to learn the words. Finally, on test trials, adults tended to look more to the target object when learning from a gaze cue, only reaching similar levels of accuracy in the nogaze condition at the end of the learning block. There was not substantial evidence, however, that the gaze manipulation influenced children's looking behavior on test trials when there was no gaze cue present, with children showing comparable and relatively low-levels of accuracy overall.

Contrary to our prediction, we did not see evidence that the gaze manipulation led children or adults to generate more accurate first shifts on test trials $\left(\beta_{\text {gaze }}=-0.50,[-1.14,0.14]\right)$ or a faster increase in first shift accuracy over the course of learning $\left(\beta_{\text {gaze*trnum }}=-0.30,[-0.74,0.12]\right)$, with the null value falling within each credible interval.

\section{General Discussion}

Does social information seeking change as a function of a speaker's helpfulness and listeners' current task goals? Here, we pursued the idea that listeners flexibly adapt their eye movements to gather social gaze when it was useful. We found that children and adults did not automatically delay their gaze shifts to seek a post-nominal gaze cue while processing familiar words. However, when processing novel words, both children and adults fixated more on a speaker to seek a post-nominal gaze cue. This delay resulted in more attention allocated to the named object and less looking to the distracter object, an effect that increased throughout the task for children. Moreover, adults, but not children, showed evidence of stronger learning in the presence of social gaze 
while both age groups were capable of learning the wordobject pairings from cross-situational statistics alone.

How should we characterize the effects of gaze in our task? Children selectively gathered social information when they were uncertain about the meaning of a new word, focusing more attention on a single object. Moreover, the presence of gaze increased children's tendency to fixate on the speaker, an increase in attention that generalized to trials without a gaze cue. This pattern of behavior shows how the presence of social information can accumulate over time, modulating the visual information that children gather during language processing. Finally, seeking social gaze increased the rate of word learning for adults. This finding dovetails with work showing how the presence of social cues can change information processing (e.g., the type of features remembered from an event) (Yoon, Johnson, \& Csibra, 2008).

This work has several important limitations. First, we did not find strong evidence that the effects of learning novel words from gaze generalized to contexts without gaze for children in Experiment 2. Moreover, children did not show robust learning of the novel word-object links overall. Prior work has shown that 3-5 year-olds learn words better from an extended, as opposed to brief, social cue to reference (Yurovsky, Wade, \& Frank, 2013). Future work could increase the length of the gaze cue, which was relatively short in these studies ( $2 \mathrm{sec}$ ) or could include a more extensive set of cues to reference such as pointing or holding objects.

Second, our experimental task measures changes in gaze dynamics in a simplified visual and linguistic context. While this approach has the benefit of providing a high degree of control, it limits our ability to generalize these results to language processing in more natural contexts. That is, in conversation, social partners can actively monitor listeners for comprehension and produce contingent responses to ensure understanding. This gap suggests two useful next steps: (1) measure changes in social information seeking within naturalistic conversations, and (2) develop lab-based methods that include contingent responding to children's actions.

These results show that even young listeners are sensitive to the informational tradeoffs in active information gathering. We found that both children and adults' decisions to seek social information varied depending on their uncertainty over word-object mappings. In the context of processing novel, but not familiar words, listeners adapted their gaze to seek a post-nominal social cue to reference. This change led to increased visual attention on a single object and less attention distributed across potential spurious word-object links. This approach sheds light on how children might integrate their prior knowledge accumulated via statistical information when deciding whether to seek information from their interlocutors during real-time language comprehension and learning.

Data/code available at https://bit.ly/2FgIbsW

E1 preregistration at https://osf.io/2q4gw/ E2 preregistration at https://osf.io/nfz85/

\section{Acknowledgements}

Thanks to K. Constandse, T. Alade, and H. Slater for help with data collection. This work was supported by an NSF GRFP to KM and a Jacobs Foundation Fellowship to MCF.

\section{References}

Baldwin, D. A. (1993). Infants' ability to consult the speaker for clues to word reference. Journal of Child Language, 20(02), 395-418.

Bloom, P. (2002). How children learn the meaning of words. The MIT Press.

Bürkner, P.-C. (2017). Brms: An r package for bayesian multilevel models using stan. Journal of Statistical Software, 80(1), 1-28.

Estigarribia, B., \& Clark, E. V. (2007). Getting and maintaining attention in talk to young children. Journal of Child Language, 34(4), 799-814.

Fourtassi, A., \& Frank, M. C. (2017). Word identification under multidomodal uncertainty. In Proceedings of the 39th annual conference of the cognitive science society.

Gibson, E., Bergen, L., \& Piantadosi, S. T. (2013). Rational integration of noisy evidence and prior semantic expectations in sentence interpretation. Proceedings of the $\mathrm{Na}$ tional Academy of Sciences, 201216438.

Hayhoe, M., \& Ballard, D. (2005). Eye movements in natural behavior. Trends in Cognitive Sciences, 9(4), 188-194.

Kelly, S. D., Özyürek, A., \& Maris, E. (2010). Two sides of the same coin: Speech and gesture mutually interact to enhance comprehension. Psychological Science, 21(2), 260267.

MacDonald, K., Marchman, V. A., Fernald, A., \& Frank, M. C. (2018). Children flexibly seek visual information during signed and spoken language comprehension.

Maris, E., \& Oostenveld, R. (2007). Nonparametric statistical testing of eeg-and meg-data. Journal of Neuroscience Methods, 164(1), 177-190.

Smith, L. B., \& Yu, C. (2013). Visual attention is not enough: Individual differences in statistical word-referent learning in infants. Language Learning and Development, 9(1), 2549.

Vigliocco, G., Perniss, P., \& Vinson, D. (2014). Language as a multimodal phenomenon: Implications for language learning, processing and evolution. The Royal Society.

Yoon, J. M., Johnson, M. H., \& Csibra, G. (2008). Communication-induced memory biases in preverbal infants. Proceedings of the National Academy of Sciences, 105(36), 13690-13695.

Yurovsky, D., Case, S., \& Frank, M. C. (2017). Preschoolers flexibly adapt to linguistic input in a noisy channel. Psychological Science, 28(1), 132-140.

Yurovsky, D., Wade, A., \& Frank, M. (2013). Online processing of speech and social information in early word learning. In Proceedings of the annual meeting of the cognitive science society (Vol. 35). 